\title{
Library Support for Resource Constrained Accelerators
}

\section{Brock-Nannestad, Laust; Karlsson, Sven}

Publication date:

2014

Document Version

Publisher's PDF, also known as Version of record

Link back to DTU Orbit

Citation $(A P A)$ :

Brock-Nannestad, L., \& Karlsson, S. (2014). Library Support for Resource Constrained Accelerators. Poster session presented at 7th Swedish Workshop on Multicore Computing (MCC14), Lund, Sweden.

\section{General rights}

Copyright and moral rights for the publications made accessible in the public portal are retained by the authors and/or other copyright owners and it is a condition of accessing publications that users recognise and abide by the legal requirements associated with these rights.

- Users may download and print one copy of any publication from the public portal for the purpose of private study or research.

- You may not further distribute the material or use it for any profit-making activity or commercial gain

- You may freely distribute the URL identifying the publication in the public portal

If you believe that this document breaches copyright please contact us providing details, and we will remove access to the work immediately and investigate your claim. 


\title{
Library Support for Resource Constrained Accelerators
}

\author{
Laust Brock-Nannestad and Sven Karlsson
}

Technical University of Denmark

\section{Motivation}

- Accelerators provide attractive power/performance trade offs

- Existing programming models treat them as offload devices

- We want accelerator cores as first class citizens

- Execute your application directly from the accelerator

- Spawn parallelism from accelerator

But retain convenience of a complete system: libraries, file I/O,

\section{Contributions}

- A new runtime for accelerators

- Accelerator drives execution - main() on accelerator

- Low overhead - moving C library to host keeps runtime small

- Implementation on x86-64 and ARM + Epiphany (Parallella)

- Evaluation using SPLASH-2; comparison to libgomp

- Measure impact on object code size by minimizing runtime

\section{Design}

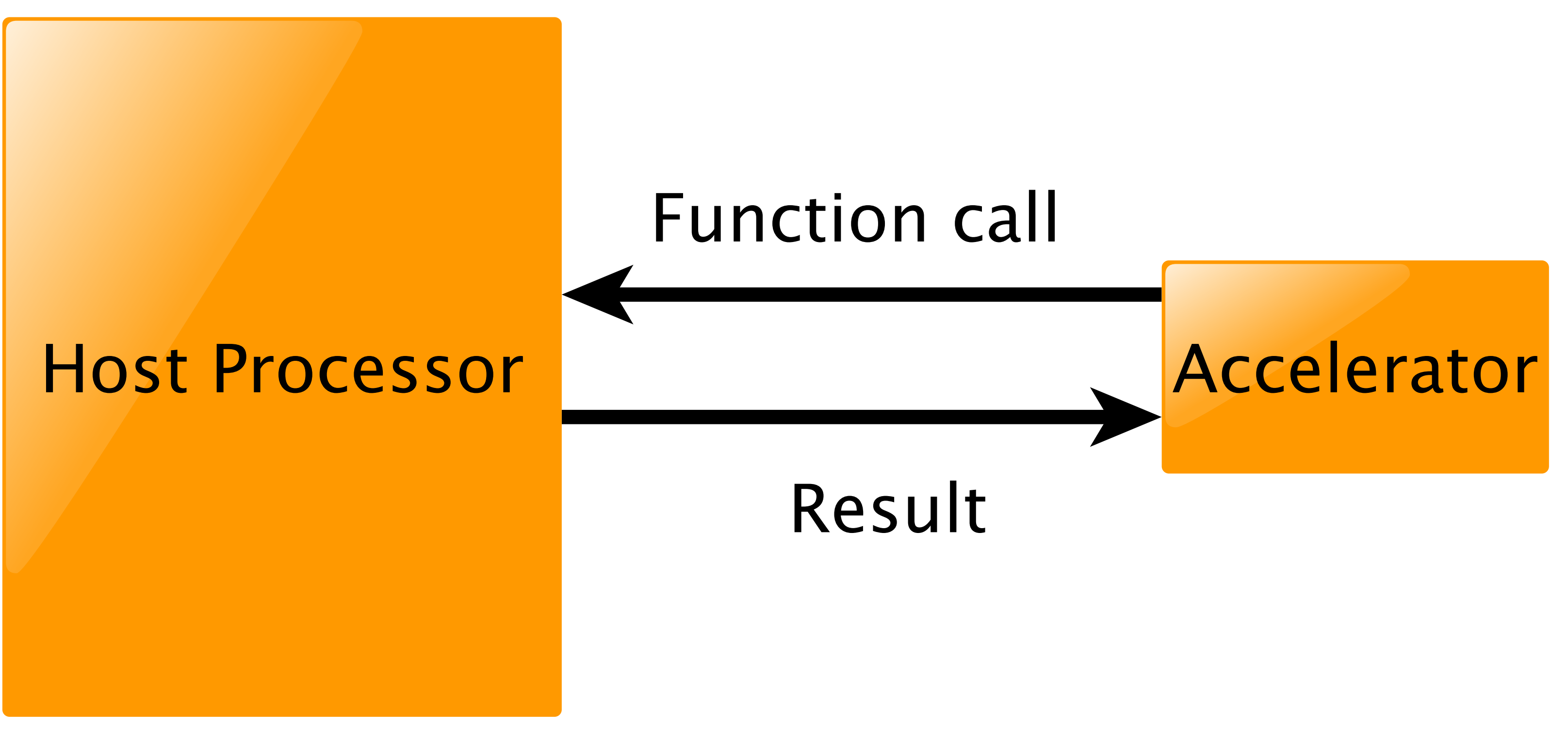

Figure : System architecture

$-\operatorname{main}() /$ master thread executes on accelerator

- Decisions on spawning parallelism taken locally

$\checkmark$ fast, low latency

- Accelerator may invoke host

- Complex decisions or function calls (C library)

- Host assists accelerator, not vice versa
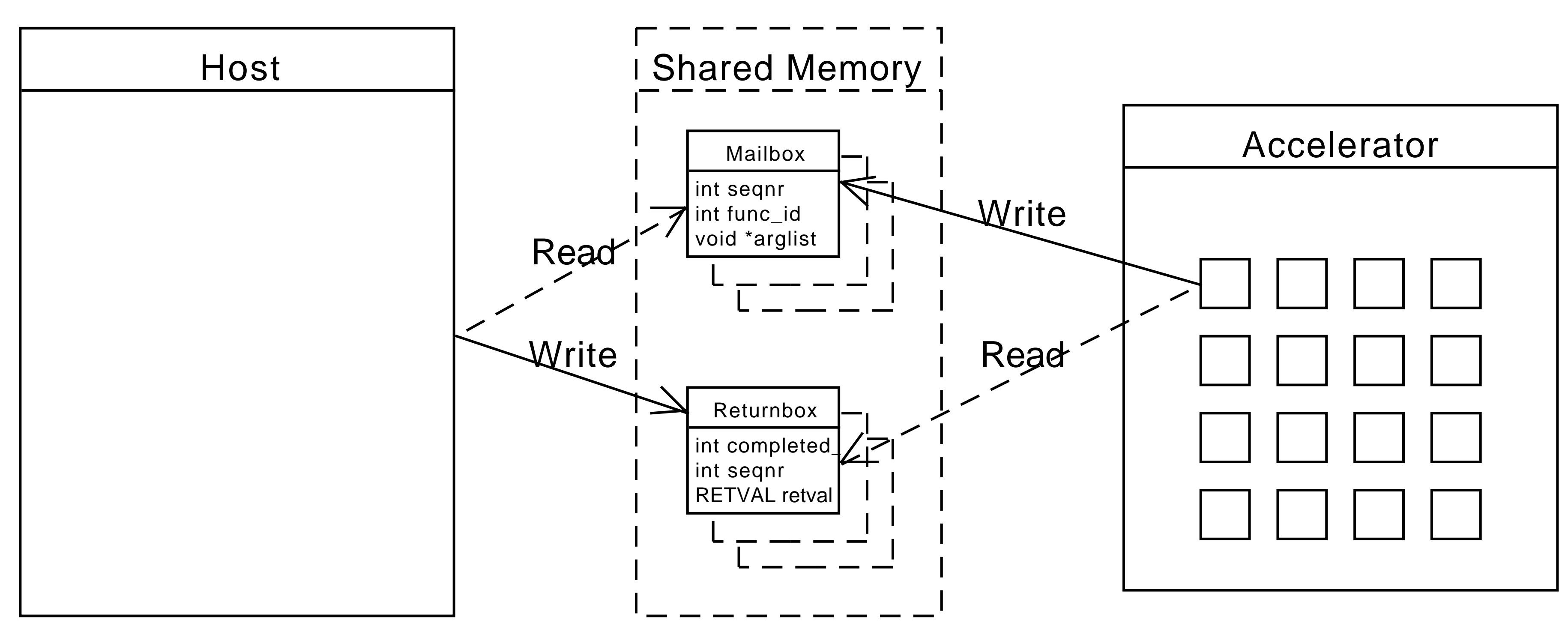

Figure : Mailboxes in shared memory

- Host and accelerator communicate through shared memory

- Mailbox data structures used for communication

Encode function call identity, arguments, and results

\section{Acknowledgments}

This work was funded by the European Artemis project nr. 295440, Portable and Predictable Performance on Heterogeneous Manycores (PaPP)

\section{Experimental setups}

-1) Dual Socket Xeon 5570 @ 2.93 GHz, Linux 3.2, GCC 4.8.1, glibc 2.17

- 2) Adapteva Parallella w. 16 core Epiphany accelerator

- SPLASH-2 benchmarks LU, FFT, C-runtime library offloaded to host

- Baseline is SPLASH-2 parallelized with GNU libgomp for GCC 4.8.1

\section{Execution time (x86-64)}

Lightweight runtime $\square$ Baseline

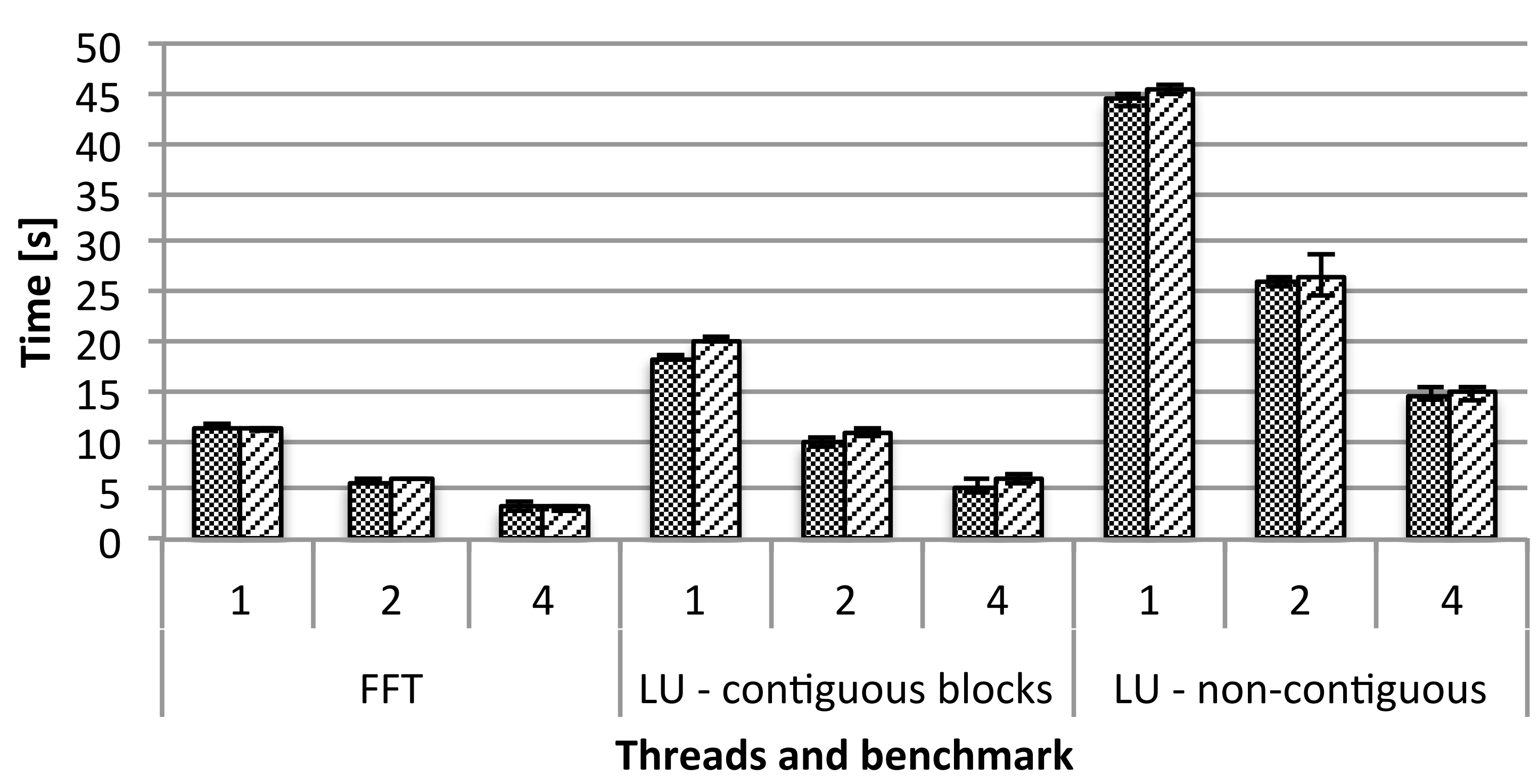

Figure : Execution time and speed up for increasing number of cores on $\times \mathbf{8 6}-\mathbf{6 4}$

\section{Object code sizes and savings}

\begin{tabular}{|l|r|c|c|}
\hline C library function (sym) & Size (bytes) & FFT & LU \\
\hline printf_fp & 9345 & X & X \\
_sin_sse2 & 8569 & $X$ & \\
-_cos_sse2 & 5159 & $X$ & \\
-getopt_internal_r & 4391 & $X$ & $X$ \\
int_malloc & 4776 & $X$ & $X$ \\
int_free & 2796 & & \\
\hline -_memset_sse2 & 2705 & & $X$ \\
malloc & 333 & $X$ & $X$ \\
(the rest) & & 271 & 565 \\
\hline Total & & $\mathbf{3 5 0 4 4}$ & $\mathbf{2 2 1 1 5}$ \\
\end{tabular}

\section{Accelerator runtime object code size}

Accelerator component x86-64 (bytes) Epiphany (bytes)

Host interface $\quad 209 \quad 96$

\begin{tabular}{l|r|r} 
Locks, Barriers & 159 & 178
\end{tabular}

$\begin{array}{lll}\text { Misc } & 130 & 228\end{array}$

Bootstrap/Initialization $\quad 202 \quad 138$

Thread creation $\quad 49 \quad 0$

\begin{tabular}{l|r|r}
\hline Total & $\mathbf{7 4 9}$ & $\mathbf{6 4 0}$
\end{tabular}

\section{Conclusions}

Object code reduction of $66-75 \%$ on benchmarks by offloading $C$ library

Low runtime footprint of 750 bytes (x86-64), 640 bytes (Epiphany)

- Computational code not influenced by remote function call overhead Performance comparable to GNU libgomp

- Initialization is single threaded. FFT initialization dominated by remote function call overhead (see paper for details)

- Future work

- Parallella/Epiphany implementation soon to be released

- Careful memory management - software caching techniques

Further information

Brock-Nannestad, L. and Karlsson, S. "Library Support for Resource Constrained Accelerators." In Improving OpenMP for Devices, Task, and More. Springer, 2014, pp.187-201. 